\title{
LA EDUCACIÓN Y LA ESCUELA DE FRANKFURT: UNA PERSPECTIVA EPISTEMOLÓGICA SOBRE LA TEORÍA CRÍTICA DE LA SOCIEDAD
}

\section{Education and Frankfurt School: An Epistemological Perspective on the Critical Theory of Society}

\author{
Karla M. Bolo-Romero \\ Universidad Femenina Sagrado Corazón \\ Lima - Perú \\ https://orcid.org/0000-0003-1494-1752 \\ kbolor@unife.edu.pe \\ Luciano C.A. Scattolon-Huapaya \\ Universidad Femenina Sagrado Corazón \\ Lima - Perú \\ https://orcid.org/0000-0002-9947-4411 \\ lucianoscattolonh@unife.edu.pe
}

\author{
Vicky I. Guevara-Granados \\ Universidad Femenina Sagrado Corazón \\ Lima - Perú \\ https://orcid.org/0000-0003-3748-374X \\ vguevarag@unife.edu.pe
}

Karina Peceros-Peceros Universidad Femenina Sagrado Corazón Lima - Perú https://orcid.org/0000-0003-1307-1361 karina.pecerosp@unife.pe

\section{RESUMEN}

Este artículo analiza los principales postulados de Max Horkheimer, exponente principal de la Escuela de Frankfurt y de la teoría crítica de la sociedad, con la finalidad de presentar una perspectiva epistemológica que compare las posiciones críticas de la sociedad de nuestros tiempos y la que concibió la Escuela de Frankfurt. Mostramos los resultados cualitativos de la encuesta realizada a 16 profesionales con título de Licenciado o grado de Magister de universidades particulares y nacionales del Perú en torno a sus concepciones sobre Epistemología.

\section{Palabras clave:}

Escuela de Frankfurt, teoría crítica, patología social, capitalismo, libertad social

\begin{abstract}
This article analyzes the main postulates of Max Horkheimer, main exponent of the Frankfurt School and of the critical theory of society, in order to present an epistemological perspective that compares the critical positions of the society of our times with the one conceived by the Frankfurt School. We show the qualitative results of a survey carried out on 16 professionals with a bachelor's or master's degree from private and national universities of Peru regarding their conceptions about Epistemology.
\end{abstract}

\section{Keywords:}

Frankfurt School, critical theory, social pathology, capitalism, social freedom 


\section{INTRODUCCIÓN}

La epistemología está orientada hacia la búsqueda y contrastación de hechos verificables que puedan ser validados en un determinado contexto; sea este cambiante o no. Esta disciplina se ha desarrollado en torno a la búsqueda del conocimiento como necesidad imperante en el ser humano, puesto que tenemos la idea de que, si conocemos la verdad, entonces podemos dominar la naturaleza. Tal vez porque este sea un deseo natural en ser humano.

Los seres humanos desde siempre han querido saber, han querido comprobar qué es aquello que saben, reconocer cuáles son las fuentes del conocimiento, y en este proceso pasamos de las ideas platónicas a las teorías de la Escuela de Frankfurt; pues se ha buscado la forma de comprobar los hechos, ya sea través de los sentidos, la razón, la experimentación, el noúmeno, la epojé, etc.

Desde el siglo XVI, nace un proceso no homogéneo, en el que la sociedad empezó a industrializarse, y en el que las sociedades se centraron en la producción y generación de riqueza, quienes ejecutaban procesos liderados por los capitales. La población en alguna medida solo ejecutaba sus labores, comprendiesen o no el devenir histórico.

Posteriormente, encontramos que en el afán de comprender qué es aquello que sucede en la sociedad la Escuela de Frankfurt genera una crítica a la postura capitalista y a las luchas de clases en el siglo XIX, así como en sus diferentes evoluciones de pensamiento, señala y critica la contradicción que existe entre la opresión del capitalismo y sus posibilidades liberadoras; es decir, hay un enfrentamiento ideológico entre el concepto de libertad y el de ciencia. En tal sentido, la teoría crítica de la sociedad se basa en una actitud reflexiva, que analiza la conducta de la sociedad de manera crítica, y cómo el capitalismo representa una opresión tanto a nivel económico, social y político.

Al respecto, Sembler (2019) señala que una de las principales preocupaciones de la Teoría Crítica tiene que ver con el desarrollo de las relaciones asimétricas o de poder en las sociedades. Cabe decir, cuando una persona tiene autoridad sobre otra, no hay igualdad. Observamos la crítica reflexiva, analizando las condiciones sociales presentes que son inducidas por procesos de dominación. Su objetivo es que el sujeto pueda liberarse de esta dominación.

Sin embargo, con el desarrollo exponencial de los medios de comunicación y la globalización, las industrias culturales adquieren un enorme protagonismo en la conformación de cosmovisiones y en la definición de la cultura. Su principal aliado es la diversión, la utilización ociosa del tiempo libre con el único objetivo de distraer, de hacer escapar al hombre de su realidad cotidiana y de devolverlo a la misma sin haber modificado ni profundizado sus categorías de entendimiento. La cultura deviene en entretenimiento y diversión (Haidar, 2016).

La teoría crítica de la sociedad concibe una nueva explicación sobre la adquisición de conocimiento, puesto que menciona la imposibilidad de aferrarse a la realidad por la fuerza del pensamiento; no puede ser inmóvil, debe ser transformadora de la realidad, y rechazar las utopías del futuro. Hay una mediación entre los procesos sociales, históricos y económicos que impactan en los conocimientos, por lo que no hay un monismo metodológico. La 
realidad no debe ser solamente teorizada, hay intereses que las parcializan; además la dicotomía de sujeto-objeto no puede mantenerse radicalmente, debe haber una "praxis social" en la que todos se inserten como seres humanos racionales. De ahî que la investigación social no se disuelve en estudios especializados, sino que la sociedad debe investigarse como un todo. La objetividad humana implica juicios de valor, por lo que, al ponerla al servicio del ser humano, deriva en una praxis liberadora: la realidad sociohistórica no puede ser justificada por ser irracional, injusta y opresora.

Durán Migliardi, (2018) con respecto al monismo metodológico señala que, en la teoría tradicional, se promueve una sola metodología de investigación. El único conocimiento riguroso y lógico es el que es vinculado con las ciencias naturales, y que se obtiene a partir de la observación de los fenómenos y que se somete a un proceso de cuantificación. El objetivo último que se busca es el dominio de la naturaleza, pues no debemos olvidar que el ser humano quiere transformar la realidad. Las ciencias humanas y sociales se elaboran teniendo como modelo a la física-matemática, perdiéndose así el sentido histórico de los acontecimientos socio-antropológicos y desembocando en el fetichismo de los hechos.

Para la teoría crítica la realidad surge de un complejo proceso de interacción entre diversos elementos; por lo que, no puede ser abordada desde un solo punto de vista. Los hechos sociales nunca están dados naturalmente, su momento presente siempre es resultado de los precedentes. Las diversas estructuras que configuran a la sociedad (la económica, la política) son fruto de intereses históricos específicos que han logrado imponerse. Por eso, los hechos actuales no son la verdad y se trata precisamente de rechazar la facticidad, lo existente.

La Escuela de Frankfurt se fundó en 1923, la misma fecha en que se publican dos importantes obras dentro de la literatura marxista: Historia y Conciencia de Clase de Georg Lukács y Marxismo y Filosofía de Karl Korsch. Lukács realiza una lectura hegeliano marxista de la dialéctica, reintroduciendo la filosofía, el movimiento negativo de las categorías, la crítica inmanente, etc., dentro de los debates marxistas. Estás últimas serán la savia teórica, el numen filosófico de la Escuela de Frankfurt.

Antes de 1923 los debates centrales dentro del marxismo se basaban en discusiones sobre el fin del capitalismo desde una perspectiva más económica. La filosofía había pasado a un segundo plano. Mucho de los aspectos críticos de la dialéctica y de la filosofía de Hegel habían pasado a ser tachados de idealistas, por lo que la reintroducción que realizó Lukács fue sumamente pertinente. No se trataba del fin del capitalismo, sino de las categorías que se movían para hacer funcionar el capital. La crítica no se trataba de partir de lo meramente empírico, sino de comprender las categorías que daban sentido y encuadraban esa ciencia desde donde existía lo empírico. La crítica inmanente, además, fue otra de las tesis centrales de Lukács, esta consistía en comprender el todo como una mediación que se desarrolla desde otra, por lo que la crítica se realizaría desde el interior de esta tesis. Los mayores recepcionadores de esta crítica fueron miembros de la Escuela de Frankfurt.

Sin embargo, esto no siempre sucedió así. La Escuela de Frankfurt tuvo muchas etapas. En su primera etapa se intentará desarrolla una sociología crítica de la 
sociedad, sin embargo, no será tan fecundo hasta el momento en que Max Horkheimer asume la dirección del instituto en 1930. Aunque durará poco, hasta 1933, debido al embate del régimen nazi, la escuela pasó a ser un centro gravitacional de distintos marxistas heterodoxos que se abrían a dialogar el marxismo con distintas filosofías de la época. Erich Fromm abordó a Marx y Freud, Herbert Marcuse a Marx y Heidegger, Adorno a Marx y Kant, etc. La Escuela tuvo que cerrar y retomará sus investigaciones en Alemania en 1950 después de la segunda guerra mundial.

La denominación de teoría crítica, si bien teje sus andamiajes en Hegel, Marx y Lukács, es explicitada por primera vez por Max Horkheimer en su Teoría tradicional y teoría crítica de 1937. En ese libro se aborda con mayor profundidad y detenimiento la diferencia entre la forma de concebir la sociedad de manera empírica y, de manera muy distinta, desde la ciencia negativa o teoría crítica. El núcleo de la teoría crítica está basado en la crítica inmanente; es decir, desarrollar una crítica no desde el exterior de una tesis, sino de buscar la negatividad interna dentro de la tesis, y desde esta misma buscar su negación y su superación. Esto estaba formulado por Hegel en su Fenomenología del Espíritu, pero también fue desarrollado por Marx en diversas obras, y de hecho en Historia y Conciencia de Clase.

Max Horkheimer siguió desarrollando estudios que fueron plasmados en dos obras fundamentales: Crítica de la razón instrumental de 1947 y Dialéctica de la Ilustración de 1944, con versión mayor de 1947, escrito junto a Theodor Adorno. En el primero libro, fiel a su crítica, desarrolla un estudio del origen de la razón y sus diferentes manifestaciones. Max Weber había desarrollado ya la idea de la razón estratégica, Marx también analizó en El
Capital como se manifiesta la "razón" capitalista en la acumulación originaria. Horkheimer verá como en Holanda se va gestando ese modo de pensar basado en la instrumentalización medio fin del ser humano. El ser humano, y en esto sigue claramente a Kant, sería un medio para la ganancia que sería el fin de toda la existencia. La razón instrumental se desarrolla a su máxima expresión en el capitalismo. Una imagen ejemplifica muy bien la idea. Un sujeto se encuentra en un árbol, él es muy bueno dominando la técnica y los instrumentos para generar mayor riqueza, ganancia, ingresos, etc., por lo que decide cortar una rama, para así poder venderla y lograr su fin. Sin embargo, él mismo está parado sobre esta rama, pero no le importa ni él mismo, sino las ganancias que obtendrá. Este sujeto termina serruchando la rama donde está parado, por lo que termina cayendo. Del mismo modo sucede con la razón instrumental, ya que esta llevaría al fin de la humanidad, a grandes cataclismos ecológicos, a grandes catástrofes si se logra el fin de la ganancia, de la obtención de mayor capital.

Finalmente, en Dialéctica de la Ilustración se plantea, otra vez desde Kant, pero principalmente desde Hegel, un intento de leer la modernidad y su expresiones tecnológicas y estéticas. La modernidad es gestada basada en la razón ilustrada, donde predomina el individuo, la mayoría de edad, la emancipación de la individualidad de los individuos. La razón es la base y la llave para lograr esa mayoría de edad, esa emancipación del ser individual. En ese sentido, la razón debe llevar al progreso del individuo. Este progreso se desarrolla con la ciencia, con la tecnología, con la mayor exaltación de la razón. La ciencia pasa a legitimar el desarrollo y viceversa, la razón lleva a una mejor forma de vida y civilización, donde todos pueden ser libres. El proyecto 
ilustrado eleva a la razón y todas sus expresiones como el centro del universo. A esto, por último, se intentará contraponer la estética. A esta totalización de la razón, a esta razón instrumental moderna, a esta centralización de la razón, se opone una estética que no está subsumida en la instrumentalidad total. La estética puede escapar a este proceso de valorización, de instrumentalización. En este sentido se apuesta por las vanguardias de inicios del siglo XX que en su forma de expresión y su fácil variabilidad no logran ser atrapados por la compra y venta de todo.

\section{Marco conceptual}

La escuela de Frankfurt toma como fuentes de pensamiento a las teorías de Weber, por el análisis del racionalismo occidental sobre el capitalismo, el estado moderno, la racionalidad científica secular, a la cultura y religión; las teorías de Freud, sobre el descubrimiento del subconsciente, de las bases del autoritarismo, del comportamiento social irracional, y la búsqueda maternalista de Edipo; la crítica del positivismo, como expresión del conformismo, a nivel ideológico y filosófico; y la teoría Marxista, a lo burgués, a la alienación del trabajador, el materialismo histórico, la historia de la lucha de clases y explotación del trabajador a lo largo de la historia.

Se orienta a la búsqueda de una transformación social, la cual sólo es posible si el hombre toma consciencia de su racionalidad; a la emancipación de los individuos, por lo que cada uno es responsable de la consecución de su libertad; frenar las injusticias sociales y el respeto por las normas morales; ya que sin ellas nos deshumanizaríamos, y los beneficios sociales que el Estado asume al tener la responsabilidad de hacerse cargo de los individuos.
Critica el análisis lógico-matemático de la ciencia, y señala la complejidad de los fenómenos sociales que trascienden estos; la reificación en las relaciones sociales (la no consciencia del ser humano); dado que la ciencia es un medio para la producción de valores sociales, además de la importancia de la ciencia de ser una fuerza productiva que hace posible el sistema industrial; denunciando la mercantilización de la ciencia en los diferentes aspectos de la sociedad.

Con respecto a la teoría crítica debemos comprender que busca descubrir la causa de los males sociales y hacerlo mediante la crítica socio filosófica; se dedicaron a investigar la cusa de los mismos. Su finalidad nunca fue el dominio del mundo, sino la liberación personal y social. Defendían la idea de que las causas últimas de los procesos sociales y políticos tenían un carácter irracional basado en miedos y deseos que condicionaban el comportamiento colectivo sin que hubiera una explicación racional para esta influencia. Estos elementos injustos y alienantes no permiten la emancipación de los seres humanos (Durán Migliardi, 2018).

En el afán de conocer la realidad, el ser humano termina separándose de la realidad y de los conocimientos que a ella le pertenecen. El hombre tiende a distanciarse de la realidad o estructura social construida por él mismo convirtiéndose en objeto y no en sujeto de acontecimientos, por tanto, deja que la realidad decida por él, incluso hasta convertirse en algo

\section{Procedimientos metodológicos}

A través de una reflexión crítica, la escuela de Frankfurt critica los males que aquejan a la sociedad, y que la ciencia no enfrenta sus problemas; señala que 
se basan en causas humanas y que la modernidad los oculta.

Realiza una reflexión crítica sobre las circunstancias y problemas de la sociedad por lo que propone mostrar y poner en evidencia estas dificultades y desigualdades a través de la crítica socio - filosófica, y planteando si es posible su corrección, la manera de lograr la satisfacción y buscar las insatisfacciones en las instituciones planteadas, buscando causas y factores, denominadas como patologías sociales, que afectan y atentan la libertad del ser humano y su dignidad, como la humillación, exclusión, cosificación, dominación, consumismo, entre otras; las cuales violentan al individuo y generan mucho malestar. Del mismo modo, identifica los mecanismos de control social y dominación establecidos por los procesos de vigilancia, así como la insatisfacción y el agravio de las poblaciones por medio de la experiencia social como proceso de generación de conocimiento.

\subsection{Fundamentos teóricos}

La escuela de Frankfurt tiene un enfoque interdisciplinario y base en el marxismo crítico, el psicoanálisis Freudiano, la filosofía crítica del siglo XIV. En tal sentido, en sincronía con el pensamiento de Weber, orientada al aprendizaje social, y que las sociedades pueden mejorar ya que aprenden socialmente. Las sociedades y sus instituciones pueden aprender a corregir estas patologías para que los individuos puedan orientarse a su libertad. Así como la importancia de la realización de la libertad de los ciudadanos, como gran valor de la modernidad, como señala Hegel.

La propuesta de la teoría crítica es lograr que la sociedad tenga libertad individual y social; recuperar la liberación histórica de los ciudadanos; sin embargo, estas no están ayudando. La sociedad cínica, de la cual formamos parte, genera daño, exclusión e impactan en su futuro. A través de esta teoría se busca que haya una orientación que permita llegar a la libertad individual y social para la satisfacción de la sociedad.

Martín (2013) con respecto a los fundamentos teóricos de la Escuela de Frankfurt señala que lo más importante es comprender el sentido liberador que debe tener la educación. La finalidad general de la práctica educativa es la superación de la barbarie, entendida como un estado de civilización técnica altamente desarrollada unido a una humanidad poseída por un impulso autodestructivo.

La única manera de lograrlo es fomentar la autorreflexión crítica y la autonomía de los estudiantes. Se trata de luchar frente a un sistema educativo, que muchas veces lo que favorece es el conformismo y la adaptación de los estudiantes. Es obvio que, para ellos, resulta más fácil y conveniente que alguien piense por ellos y les diga lo que tienen que hacer. Por lo que, es necesario que el docente forme a sus alumnos en la resistencia y en la contradicción, formar tanto el pensamiento como la voluntad. Es tarea del docente buscar y lograr que sus alumnos, se alejen de la influencia de los prejuicios y supersticiones y aprendan a pensar por sí mismos, haciendo uso de su razón de manera libre. Esta es la llamada emancipación intelectual que va a permitir obtener la humanidad en uno mismo.

Con esta concepción de la educación, en oposición al docente ejecutivo de la tradición positivista-conductista, en la que el docente es reflejo de un sistema 
político despótico; el estudiante deja de ser un instrumento dócil controlado por los poderes de la sociedad, deja de ser un agente pasivo que sólo se limita a recibir y repetir de memoria la información que recibe del profesor, que se ve resignado a adaptarse y a aceptar todo lo que le dicen; para convertirse en un estudiante que toma conciencia de sí mismo, tanto en la teoría como en la práctica, en un estudiante que dialoga, que problematiza el contenido; es decir, en un estudiante que razona.

En conclusión, el enfoque del docente emancipador de la Escuela de Frankfurt cuestiona el modelo ejecutivo de docente basado en la racionalidad cientificista y rechaza la idea de un sistema educativo que esté al servicio de las ideologías dominantes donde se vea como simples objetos a los seres humanos. Para el enfoque Frankfurtiano, el docente debe entender que su labor en el aula se basa principalmente en formar estudiantes que sean capaces de luchar por la justicia y su libertad.

\subsection{Paradigmas y elementos instrumentales}

En base a las patologías sociales que dañan a la sociedad, como el impacto del capitalismo que ha avanzado en todo el mundo, la escuela de Frankfurt busca asumir una postura ética y política adecuada que permita primero poner en evidencia los problemas que la aqueja y luego la búsqueda de sus soluciones (Rodríguez Reyes, 2017).

Pretende que el conocimiento científico y tecnológico tenga una aplicación ética que permita dar respuesta a los problemas de investigación que la sociedad necesita, poniendo al descubierto estos males, y que permita lograr la libertad de los individuos.

Al realizar un análisis de la problemática en diversos niveles de profundidad, se puede identificar sus causas ocultas, y establecer mecanismos de resolución para orientar al aprendizaje social, y que sus instituciones puedan velar por estos valores y libertad.

\section{Metodología}

La metodología utilizada para la realización del trabajo en cuestión fue de enfoque cualitativo, puesto que se analizaron categorías como: epistemología, importancia de la epistemología, dificultades del investigador, estrategias para desarrollar una perspectiva epistemológica deseable y perfil del investigador. La población que se entrevistó fue un total de 16 profesionales y la muestra fue probabilística aleatoria coincidente con la población. Todos los entrevistados son egresados de las carreras de Educación y Humanidades. El $75 \%$ cuenta con el grado de Magister y el $25 \%$ con el grado de bachiller.

Para analizar los resultados de las entrevistas se utilizó el razonamiento inductivo (de lo particular a lo general) y deductivo (de lo general a lo particular), de manera secuencial para procesar las respuestas en torno a las categorías mencionadas. Primero, se realizó la recolección de las respuestas; luego se analizaron las percepciones sobre el tema cuestionado para desarrollar un diseño colectivo y contrastar los resultados frente a la teoría propuesta por la Escuela de Frankfurt. 


\section{Resultados y discusión}

TABLA N ${ }^{\circ} 1$ Entrevista investigadores en educación

\begin{tabular}{|c|c|c|c|c|}
\hline \multicolumn{2}{|c|}{ Docente } & Especialidad & Sexo & $\begin{array}{c}\text { Tipo de } \\
\text { institución }\end{array}$ \\
\hline 1. & Milagros & $\begin{array}{l}\text { Bachiller en Educación } \\
\text { Licenciada en Educación } \\
\text { Maestra en Educación - Docencia e } \\
\text { investigación universitaria }\end{array}$ & Femenino & Pública \\
\hline 2. & Nora & $\begin{array}{l}\text { Licenciada en Humanidades } \\
\text { Maestra en Educación } \\
\text { Doctoranda Educación }\end{array}$ & Femenino & Pública \\
\hline 3. & Linda & $\begin{array}{l}\text { Bachiller en Educación } \\
\text { Licenciada en Educación } \\
\text { Maestra en ciencias de la Educación } \\
\text { Evaluación y acreditación de la calidad } \\
\text { educativa }\end{array}$ & Femenino & Pública \\
\hline 4. & Piera & $\begin{array}{l}\text { Bachiller en Administración } \\
\text { Maestría en Educación }\end{array}$ & Femenino & Pública \\
\hline 5. & Isaac & $\begin{array}{l}\text { Bachiller en derecho y ciencias políticas } \\
\text { Abogado }\end{array}$ & Masculino & Pública \\
\hline 6. & Gonzalo & $\begin{array}{l}\text { Bachiller en humanidades con mención } \\
\text { en filosofía }\end{array}$ & Masculino & Particular \\
\hline 7. & Vilma & $\begin{array}{l}\text { Bachiller en educación } \\
\text { Licenciado en Educación }\end{array}$ & Femenino & Pública \\
\hline 8. & Paúl & $\begin{array}{l}\text { Bachiller en ingeniería de sistemas } \\
\text { Ingeniero de sistemas } \\
\text { Magister en docencia universitaria } \\
\text { maestro en ingeniería de sistemas con } \\
\text { mención en tecnologías de la información }\end{array}$ & Masculino & Particular \\
\hline 9. & Joel & $\begin{array}{l}\text { Bachiller en Educación } \\
\text { Licenciado en Educación } \\
\text { Magíster en Educación } \\
\end{array}$ & Masculino & Pública \\
\hline 10. & Lía & $\begin{array}{l}\text { Bachiller en Filosofía } \\
\text { Licenciada en Filosofía }\end{array}$ & Femenino & Pública \\
\hline 11. & Flavio & $\begin{array}{l}\text { Bachiller en teología } \\
\text { Maestro en Sagrada Teología con men- } \\
\text { ción en teología dogmática }\end{array}$ & Masculino & Privada \\
\hline 12. & Eduardo & $\begin{array}{l}\text { Bachiller en Educación } \\
\text { Bachiller en teología } \\
\text { Licenciado en teología } \\
\text { Maestro en Sagrada Teología con men- } \\
\text { ción en teología dogmática } \\
\end{array}$ & Masculino & Privada \\
\hline 13. & Andrea & $\begin{array}{l}\text { Bachiller en Educación Inicial. } \\
\text { Licenciada en Educación Inicial. }\end{array}$ & Femenino & Privada \\
\hline 14. & Pamela & $\begin{array}{l}\text { Bachiller en Educación Inicial } \\
\text { Licenciada en Educación Inicial. }\end{array}$ & Femenino & Privada \\
\hline
\end{tabular}




\begin{tabular}{l|l|l|l|l}
\hline 15. & Stefany & $\begin{array}{l}\text { Bachiller en Educación. } \\
\text { Licenciada en Educación. } \\
\text { Maestría en Ciencias de la Educación } \\
\text { con mención en gestión y administración } \\
\text { educativa }\end{array}$ & Femenino & Pública \\
\hline 16. & Janike & $\begin{array}{l}\text { Bachiller en Educación } \\
\text { Licenciada en Educación Secundaria } \\
\text { especialidad en Matemática y Física }\end{array}$ & Femenino & Pública \\
\hline
\end{tabular}

Tabla $\mathrm{N}^{\circ}$ 2: Análisis de la categoría 1

\begin{tabular}{|c|c|}
\hline Categoría 1 & Sobre idea de epistemología \\
\hline Tipo de respuesta & Contenido de respuestas \\
\hline $\begin{array}{l}\text { Tipo de respuesta } 1 \\
\text { Crítica (análisis preciso) } \\
8 \text { respuestas } \\
50 \%\end{array}$ & $\begin{array}{l}\text { - Es la filosofía de la ciencia que estudia cómo se } \\
\text { construye el conocimiento científico. } \\
\text { - Es una parte de la filosofía que estudia el co- } \\
\text { nocimiento científico, su naturaleza, alcances y } \\
\text { fundamentos. } \\
\text { - La epistemología reviste una gran importancia en } \\
\text { el campo educativo porque la práctica educativa } \\
\text { tiene que fundamentarse en bases científicas sóli- } \\
\text { das, en teorías educativas verificadas, confiables y } \\
\text { que llevarán a una mejora en el campo educativo. }\end{array}$ \\
\hline $\begin{array}{l}\text { Tipo de respuesta } 2 \\
\text { Utilitaria (Análisis para un fin) } \\
6 \text { respuestas }\end{array}$ & $\begin{array}{l}\text { - Es importante para definir el marco conceptual y } \\
\text { luego aplicarlo. } \\
\text { - La investigación educativa, es indispensable no } \\
\text { solo por el cuestionamiento para establecer los } \\
\text { procesos correctos para crear conocimiento, sino } \\
\text { en revisar el producto de las investigaciones en el } \\
\text { campo educativo. } \\
\text { - Es muy importante, ya que va a ayudar a seguir } \\
\text { estudiando las novedades educativas para com- } \\
\text { prender la capacidad y el funcionamiento del } \\
\text { conocimiento de los alumnos y ver sus límites, } \\
\text { para luego aplicar una nueva metodología que } \\
\text { los ayude. }\end{array}$ \\
\hline $\begin{array}{l}\text { Tipo de respuesta } 3 \\
\text { Imprecisa (análisis confuso) } \\
2 \text { respuestas }\end{array}$ & $\begin{array}{l}\text { - La epistemología se encarga de estudiar los medios } \\
\text { que permiten la adquisición de conocimiento. } \\
\text { - Teniendo en cuenta que la educación es todas } \\
\text { aquellas acciones de investigación para poder } \\
\text { mejorar siempre cada uno de los procesos de } \\
\text { aprendizaje. }\end{array}$ \\
\hline
\end{tabular}

Elaboración propia 
Tabla N 03 - Análisis de la Categoría 2

\begin{tabular}{|c|c|}
\hline Categoría 2 & Sobre las dificultades del rol en la investigación \\
\hline Tipo de respuesta & Contenido de respuestas \\
\hline $\begin{array}{l}\text { Tipo de respuesta } 1 \\
\text { Impacto económico } \\
4 \text { respuestas }\end{array}$ & $\begin{array}{l}\text { - El acceso a la información de calidad porque tiene } \\
\text { costos muy altos } \\
\text { - No se asignan los presupuestos necesarios a la inves- } \\
\text { tigación en el campo educativo porque las entidades } \\
\text { responsables no les dan la prioridad que necesitan. }\end{array}$ \\
\hline $\begin{array}{l}\text { Tipo de respuesta } 2 \\
\text { Impacto social } \\
4 \text { respuestas }\end{array}$ & $\begin{array}{l}\text { - Tiene dificultades al establecer el nivel de participación } \\
\text { y al abrir nuevas experiencias que generen cambios en } \\
\text { sus construcciones mentales y que tengan un impacto } \\
\text { real en solucionar problemas de la sociedad. } \\
\text { - En educación en el Perú, la investigación es más de } \\
\text { escritorio, no se profundiza y se tienen que seguir } \\
\text { parámetros y criterios metodológicos que no permiten } \\
\text { innovar. }\end{array}$ \\
\hline $\begin{array}{l}\text { Tipo de respuesta } 3 \\
\text { Impacto académico } \\
9 \text { respuestas }\end{array}$ & $\begin{array}{l}\text { - La falta de información previa necesaria. Los estudios } \\
\text { realizados son carentes de conocimiento que permitan } \\
\text { orientarse al objetivo del investigador. } \\
\text { - Acceso a plataformas de investigación para obtener } \\
\text { una información confiable. }\end{array}$ \\
\hline
\end{tabular}

Elaboración propia

Tabla Nº4 - Análisis de la Categoría 3

\begin{tabular}{|c|c|}
\hline Categoría 3 & Sobre las estrategias de perspectiva epistemológica \\
\hline Tipo de respuesta & Contenido de respuestas \\
\hline $\begin{array}{l}\text { Tipo de respuesta } \mathbf{1} \\
\text { Estrategias de compren- } \\
\text { sión } \\
6 \text { respuestas }\end{array}$ & $\begin{array}{l}\text { - Se necesita definición y claridad en los conceptos, dado } \\
\text { que la manera cómo se enseña no permite recordarlos. } \\
\text { - Consultar fuentes de información de autores vigentes } \\
\text { para comprender los elementos que propician cono- } \\
\text { cimientos. }\end{array}$ \\
\hline $\begin{array}{l}\text { Tipo de respuesta } 2 \\
\text { Estrategias de Elabora- } \\
\text { ción } \\
3 \text { respuestas }\end{array}$ & $\begin{array}{l}\text { - Primero adquirir información relevante al estudio a } \\
\text { realizar. Realización de estadística como apoyo. } \\
\text { - Brindar talleres de diseño metodológico, a los docentes, } \\
\text { para que, sobre las líneas de investigación de la univer- } \\
\text { sidad, cada uno brinde sus propuestas o alternativas de } \\
\text { diseño para ser desarrollados, de esa forma, realicen el } \\
\text { efecto multiplicador en sus estudiantes y haya mayores } \\
\text { y mejores perspectivas para abordar una temática. }\end{array}$ \\
\hline $\begin{array}{l}\text { Tipo de respuesta } 3 \\
\text { Estrategias de organi- } \\
\text { zación } \\
8 \text { respuestas }\end{array}$ & $\begin{array}{l}\text { - Actualizarse constantemente, revisar diferentes artí- } \\
\text { culos recientes, revisar diferentes contenidos. } \\
\text { - Tener una biblioteca o cursos bien ordenados que per- } \\
\text { mitan acceder a la literatura a modo de guía: de esta } \\
\text { manera se evita que los investigadores se pierdan en } \\
\text { gigantesca variedad de información que hay. }\end{array}$ \\
\hline
\end{tabular}

Elaboración propia 
Tabla Nº5 - Análisis de la Categoría 4

\section{Categoría 4 \\ Perfil epistemológico de todo estudiante y do- cente}

\section{Tipo de respuesta}

Tipo de respuesta 1

Orientación metodológica

\section{Contenido de respuestas}

- El docente debe tener un sistema de creencias orientado a la calidad en la educación y separar sus ideologías al momento de realizar la investigación

- Sobre cualquier cualidad debe permanecer un espíritu crítico que sepa pensar con constantes embates, es decir, que esté dispuesto a equivocarse en sus convicciones para acceder a más conocimiento, y de mejor calidad.

Tipo de respuesta 2 Orientación innovadora

- Arriesgarse a salirse de las investigaciones tradicionales.

- Capacidad de relacionarse con los demás (buena comunicación). Estar dispuesto al cambio

- Ir en busca de la verdad. Cuestionar el porqué de la realidad que tenemos. Ya sea un objeto de estudio o un resultado cuantitativo

- Amplias habilidades de comprensión lectora y reflexión crítica.

\section{Análisis de la categoría 1}

La primera pregunta de la entrevista aborda dos temáticas: el concepto y la importancia de epistemología. Se ha clasificado las respuestas en tres tipos: críticas, utilitarias e imprecisas. Críticas porque abordan un conocimiento previo y preciso de ambas temáticas; utilitarias, puesto que implican de manera directa el hecho educativo y; finalmente, imprecisas porque se alejan de la respuesta.

\section{Respuesta crítica}

El $50 \%$ de los entrevistados formula una respuesta de tipo crítica, observamos que tienen una idea especifica de qué es epistemología, la comprenden como una teoría de la ciencia y también comprenden porque ésta es importante en el desarrollo de la sociedad. Además, reconocen que la epistemología es importante para el desarrollo de la educación. Desde esta perspectiva podemos inferir que existe cierta confiabilidad en la posibilidad de incrementar o de ampliar el plano educativo vinculándolo con la idea de investigar; puesto que, si contamos con una muestra intencional que señala que reconocen el funcionamiento de la investigación, así como su importancia, entonces la probabilidad de mejorar en las aulas universitarias es posible. Cabe señalar que 6 de los 8 entrevistados que respondieron de manera crítica pertenecen a universidades nacionales; por tanto, debemos obviar en algunos casos ciertos clichés con respecto al avance y/o desarrollo de esta ciencia en las aulas nacionales.

\section{Respuesta utilitaria}

En cuanto a este tipo de respuesta cabe señalar que el 37\% nos brindó una 
alternativa utilitaria en torno al concepto e importancia de epistemología, puesto que señalaron que a partir de ella se puede comprender cuáles son las novedades en el plano educativo, así avanzar y ofrecer un marco conceptual más amplio que brinde diversas posibilidades en el campo de la investigación. Debemos resaltar el hecho de que en esta respuesta encontramos cierta parcialidad en cuanto a la procedencia académica de los entrevistados. Podemos deducir el hecho de que los entrevistados reconocen el para qué de la epistemología. Si tenemos en cuenta que la Escuela de Frankfurt ofrece mucha importancia a la utilización de la ciencia, sin temor a equivocarnos podemos decir que existen las posibilidades idóneas de proponer cambios en el ámbito educativo. Ahora bien, cabe señalar que si preguntamos en este momento cla epistemología es útil? Desde las respuestas obtenida, podemos decir que sí. ¿Podemos avizorar un cambio? Tal vez, a largo plazo y dependiendo de cómo se busquen los resultados. La idea de establecer alianzas educativas implica el hecho de ampliar el panorama de la ciencia.

\section{Respuesta imprecisa}

Hemos podido observar en el marco de discusión de las respuestas que ha habido cierta congruencia en torno a la conceptualización e importancia de la epistemología, pues ha sido relacionada al campo educativo y al hecho de mejorar la sociedad. Sin embargo, para sorpresa nuestra el $12.5 \%$ responde de manera muy imprecisa: se encarga de estudiar los medios, notamos que tal vez en el camino el entrevistado perdió el rumbo del plano epistemológico. Confunde medios con fines; sin embargo, no debe sorprendernos tanto, ya que muchas veces no prestamos atención en el logro de nuestros aprendizajes significativos, luego tenemos resultados como estos.

\section{Conclusiones generales de los tipos de respuesta de la categoría 1}

En tal sentido, en torno a las respuestas obtenidas sobre el concepto de epistemología, ésta puede ser entendida de manera precisa para algunos, utilitaria para otros e imprecisa para algunos. Asimismo, la importancia que se desprende va a depender del tipo de concepto de epistemología.

Podemos querer hacer cambios, podemos o pretendemos cambiar el mundo y abogamos por una educación de calidad, pero no podemos alejarnos de la realidad, pues nos guste o no debemos ser conscientes del hecho de que lo fundamental en la aulas universitarias es la investigación para mejorar la sociedad; entonces, si queremos realmente queremos cambiar la sociedad, debemos mejorar los cimientos de esta, esto implica el hecho de que no solamente podemos hacerlo desde las aulas universitarias si no a través de una investigación formativa desde la básica regular, lo que implica una versión más aplica de la educación y de la calidad de la misma, lo que nos confronta con la idea de Horkheimer acerca de la naturaleza de la investigación.

\section{Análisis de la categoría 2}

El rol de la investigación en la sociedad ha cobrado importancia en los últimos años, en el contexto nacional se aborda la investigación a partir del cambio de la ANR a la SUNEDU, muchos docentes universitarios se vieron en la obligación de hacer lo que no habían hecho hace muchos años: investigar. La misión de todo docente universitario debe ser procurar un cambio para la sociedad, o 
por lo menos para la institución en la que desarrolla, innovando, transformando, creando, tratando de romper paradigmas para hacer frente a una realidad social absolutamente cambiante.

La confrontación de la escuela de Frankfurt con las dificultades que tienen los investigadores nos ha develado tres tipos de respuestas, a saber: Impacto social, Impacto económico e Impacto académico. Podría pensarse que tal vez, muchos han tenido la intención; sin embargo, no han tenido el apoyo de la institución para poder desarrollar investigaciones que aporten, pues por lo general, la mayoría de los docentes está esperando las horas académicas lectivas que tendrá.

\section{Impacto económico}

Las respuestas de los entrevistados nos conducen a formular un tipo de respuesta denominado impacto económico, porque las expresiones utilizadas nos hacen conocer la falta de financiamiento que existe en nuestro país para investigar. Peor aún, la imposibilidad siquiera de acceder a algunas páginas o repositorios que el investigador tiene que pagar de su bolsillo; sin embargo, el mérito y la gloria acaba siendo para la institución. La posibilidad siquiera de financiar una investigación está basada en una serie de concursos y documentos que normalmente no se logra finalizar, puesto que algunas terminan archivadas, en el olvido o son de muy poca difusión, por lo tanto, no merecen la asignación del presupuesto puesto que no son llamativas.

El impacto económico está asociado, incluso con la asignación de horas en la carga académica docente. Puesto que, las universidades tienen claro el hecho que, para tener importancia en el mundo académico, necesitan docentes investigadores, pero los vicerrectorados de investigación no cumplen su función real, por eso encontramos en los entrevistados un sinsabor, que se refleja en su falta de compromiso para una investigación decente y coherente, que finalmente termine impactando en la sociedad.

\section{Impacto social}

La misión de toda institución educativa es transformar la sociedad, y existe una honda preocupación por los perfiles de egresos de los estudiantes, lo que implica de manera directa el tácito compromiso al que accede la institución educativa, con la formación de nuevos profesionales, es decir, no se trata sólo de repartir títulos al por mayor, pues existe un compromiso transformador de la sociedad, de la "polis", puesto que el hombre es un ser social por naturaleza, y por lo tanto, tiene que cambiar la sociedad, modificarla para hacerse en ese continuo devenir histórico; no obstante, encontramos que sólo el $25 \%$ de los entrevistados tiene clara la propuesta social de la investigación; tal vez porque se cansó, o sigue esperando el sueño de los justos, o tal vez porque siente miedo a que su trabajo quede sólo archivado, y entre el no hacer y el hacer, se decide por la inacción. Por tanto, se está perdiendo la capacidad críticotransformadora de los seres humanos.

De alguna manera observamos cierto conformismo frente a la a Educación y su sentido liberador (individual y colectivo): La finalidad general de la práctica educativa, desde la Escuela de Frankurt, es la superación de la barbarie, entendida como un estado de civilización técnica altamente desarrollada unido a una humanidad poseída por un impulso autodestructivo. Por tanto, la única manera de lograrlo es fomentar la 
autorreflexión crítica y la autonomía de los estudiantes. Se trata de luchar frente a un sistema educativo, que muchas veces lo que favorece es el conformismo y la adaptación de los estudiantes. Para el alumno, resulta más cómodo y menos esforzado que alguien piense por él y le diga lo que tiene que hacer. Por lo que es necesario que el docente forme en las ávidas mentes de sus alumnos la resistencia y la contradicción, formar tanto el pensamiento como la voluntad. Debe buscar que sus alumnos aprendan a pensar por sí mismos, que hagan libre uso de su razón, alejados de toda clase de prejuicios y supersticiones. Se trata de conseguir la emancipación intelectual dando lugar al cumplimiento de la humanidad en uno mismo.

\section{Impacto académico}

La investigación académica es muy importante, no sólo porque brinda prestigio al investigador; sino también porque puede devolver la esperanza a un país en vías de desarrollo, cuyo sector educativo ha tenido una serie inconclusa de transformaciones pero que finalmente no logró aterrizar y seguimos en la prueba constante de proyectos nacionales de educación, inacabados, inconclusos que suenan a maravilla en la documentación; pero que en la práctica sólo muestra la precariedad del sistema educativo de nuestra nación.

Hemos cambiado de proyectos educativos a lo largo de los últimos 20 años, y ninguno ha rendido fruto, porque seguimos siendo el penúltimo lugar en educación a nivel latinoamericano; y las respuestas de los investigados reflejan esta precariedad. Puesto que se señala la falta de coordinación entre pares, por ejemplo. Tal vez, por la naturaleza egoísta del ser humano, o tal vez, porque queremos la gloria personal; la falta de claridad en las líneas de investigación y el olvido en el que viven las humanidades en nuestro país, hoy en día, que finalmente nos conduce a una deshumanización. La misma, que tal vez en algún momento, nos lleve a una nueva barbarie. Hay un llamado para que las instituciones se den cuenta de que deben ampliar estas líneas de investigación y humanizarlas, para lograr una transformación real de la sociedad, para poder, desde nuestro escritorio, tener una perspectiva holística, que permita integrar los perfiles de egreso, orientados a las necesidades de la sociedad. El cambio real, está en la claridad de las políticas educativas, dispuestas a ayudar realmente al docente investigador, aquel que hace investigación cualitativa, que suma al lado humano.

Estas respuestas de los entrevistados, nos hace ver que hay un llamado crítico a las instituciones para que exista una mejor formación académica en el plano epistemológico, sin descuidar el lado humano.

\section{Conclusiones generales de los tipos de respuesta de la categoría 2}

El análisis de la categoría 2 nos muestra que el impacto se deja sentir en tres dimensiones: social, económico y académico. Observamos que, en el nivel social, las instituciones educativas no están formando investigadores para resolver y/o desarrollar propuestas sobre las problemáticas sociales, puesto que hay una visión sesgada de la epistemología y sus campos de acción. Esto implica, por tanto, que nos alejamos de los presupuestos de la escuela de Frankfurt, puesto que la investigación, desde su punto de vista, se centra específicamente en proponer soluciones a los problemas de la sociedad. En cuanto al impacto 
académico, puede observarse que más del 50\% de entrevistados, tiene una preocupación agobiante por el desarrollo de la investigación; puesto que encuentran en su camino muchas dificultades, y las mayores de estas tienen que ver específicamente con la falta de acceso a repositorios apropiados; pero costosos. Asimismo, a la revisión por pares, que se presta a diversas interpretaciones y que no permite el avance de la investigación. Finalmente, el impacto económico que obviamente implica una salida de dinero del bolsillo del propio investigador. Lo cual representa una de las mayores dificultades a enfrentar en este proceso de investigación.

\section{Análisis de la categoría 3}

Investigar implica una planificación exhaustiva, puesto que se tienen que medir u observar indicadores tales como: el tiempo, la comprensión de aquello que se va a investigar, la elaboración de la investigación y también la organización. Ante estos tipos de respuestas, los entrevistados nos dejaron ver que podíamos tipificar las siguientes estrategias: las de comprensión, las de organización y las de elaboración.

Esta categorización nos ayuda a comprender que sí existe una preocupación por la organización de los eventos que den como resultado una buena u óptima investigación. Así mismo, que existe una preocupación por la forma en la que se tiene que elaborar los pasos a seguir para estructurar la investigación.

Los investigadores señalan que se debe contar con bibliotecas. Asimismo, hacen hincapié en la palabra constancia; esto nos deja ver que de una u otra forma hay cierta preocupación por la mejora de las estrategias de la investigación. También en el manejo de conceptos, incluso, alguno de los investigadores hace un llamado de atención al Poder Ejecutivo. A partir de estas respuestas hemos podido clasificar o catalogar lo siguiente:

\section{Estrategias de comprensión}

El 37.5\% de los investigadores señala que una de las estrategias para tener en cuenta para desarrollar una óptima perspectiva epistemológica tiene que ver con la comprensión, es decir: definir los conceptos, para saber hacia dónde nos llevará la investigación; consultar fuentes de información, para poder comprender los indicadores implícitos en la investigación.

La comprensión también está ligada a la contrastación de los hechos con la realidad; a partir de estas respuestas, podemos observar, con optimismo, que existe una preocupación por contrastar los hechos con la realidad. Por lo tanto, estas estrategias permiten desde nuestra perspectiva, el paso inicial para el desarrollo de las investigaciones que resuelvan alguno de los problemas sociales de nuestra complicada realidad.

Es decir, teniendo en cuenta lo que señala la escuela de Frankfurt: tener una preocupación constante por los problemas sociales, no se trata solo de ver estos problemas, la temática central es poder encontrar la raíz de los mismos. Se trata pues de buscar cuáles son las causas de los mismos.

Los seres humanos desde siempre hemos buscado las respuestas a todo lo que sucede a nuestro alrededor, hemos buscado de manera ávida respuestas, queremos saberlo todo, tal vez sea por naturaleza como dice Aristóteles o tal vez sea porque Dios nos impulsa a eso, desde Tomas, pero lo cierto es 
que buscamos soluciones. Horkheimer prioriza la importancia de la ciencia para comprender y resolver para corregir. No debemos olvidar la temática humana en aras de la ciencia, debemos en aras de la ciencia resolver los problemas humanos.

\section{Estrategias de Elaboración}

En cuanto al tipo de respuesta: estrategias de elaboración, observamos con cierta preocupación que solamente el $18.75 \%$ de los entrevistados señala cierto interés por este tipo de respuesta; es decir: revisión estadística, bibliografía pertinente, operacionalización de las variables, talleres de diseño metodológico; por tanto, podemos inferir a partir de estas respuestas la escasa preparación que reciben los investigadores en las instituciones educativas en el marco estadístico.

En el proceso de investigación son importantes las herramientas con las que se podrá interpretar los datos que se han investigado; no obstante, a partir de las respuestas de los entrevistados, podemos inferir una carencia de estos instrumentos y a la vez podemos darnos cuenta de que el porcentaje de quienes se preocupan por estas herramientas es reducido. Tal vez, las observaciones señaladas sean las causas por las cuales, la investigación educativa no ha logrado posicionarse en nuestro país. O tal vez, porque sólo pensamos que se debe hacer investigación en el área de ciencias. Olvidando así que la investigación debe partir de los problemas humanos.

Al confrontar las respuestas de los entrevistados con los puntos neurálgicos de la Escuela de Frankfurt hacemos hincapié en el hecho de que se debe investigar cada uno de los problemas de la sociedad. Los seres humanos no podemos ser particularistas en este sentido, la ciencia no puede estar a merced de la ciencia, sino a favor del desarrollo humano. La Escuela de Frankfurt buscaba la mejora de la sociedad, se oponía al Capitalismo y a los órdenes establecidos, buscaba indagar a partir de toda la cultura, puesto que la premisa epistemológica era interpretar la sociedad.

\section{Estrategias de organización}

El 50\% de los investigados señala respuestas como: elaboración de proyectos de investigación, preocupación porque el estado a través del Poder Ejecutivo intervenga en una investigación socioformativa, desde la Educación Básica Regular para poder implementar estrategias de mejora para el área epistemológica, difusión de líneas de investigación, propuesta de temas apropiados a la coyuntura social, formación de semilleros de investigación, encuentros entre investigadores, capacitación constante, formación de equipos colaborativos, actualización constante.

Estas respuestas nos dejan entrever que sí existe una honda preocupación por la organización referente al área de investigación. Lo que implica que la revisión de los problemas de la sociedad podría resolverse a partir de una mejor estructura; sin embargo, las políticas educativas en nuestro país están lejos de tener metas objetivas. Por lo general, se han estructurado diversos proyectos, pero ninguno con metas objetivas y reales.

Lamentablemente, nuestra realidad nos muestra lo alejados que estamos de gestionar estrategias de organización que impliquen las mejoras de la sociedad o la implicancia de la tarea del investigador sobre la misma. Puesto que, en el área educativa de la Básica Regular, se han diseñado una serie de propuestas 
inconclusas. Puede que exista cierta preocupación, pero no hay objetivos o metas realistas aplicables a corto plazo, y a través del cambio y dinámica constante, no se ha logrado aterrizar las modificaciones necesarias, por la falta de políticas que lo permitan.

La Escuela de Frankfurt hace hincapié en el desarrollo de la sociedad y crítica el desarrollo de los medios de comunicación y la globalización; puesto que, desde su perspectiva se utilizaba de manera ociosa del tiempo libre. Por tanto, a partir de esta tipificación de respuesta se propone no escapar de nuestra realidad cotidiana y profundizar en todas las categorías de conocimiento.

\section{Conclusiones del análisis de la categoría 3:}

Para la Escuela de Frankfurt la sociedad, el estado y sus instituciones deben contribuir a que las personas realicen y alcancen sus proyectos para la mejora de su calidad de vida. El sistema económico debe garantizar la satisfacción de necesidades, promover al mismo tiempo un estado de derecho para todos, y si no puede hacerlo se tienen que hacer correcciones para que las personas puedan alcanzar el ideal de mejora de la sociedad. A partir de estas observaciones y de la confrontación con las respuestas de los entrevistados podemos deducir que si no existe una organización estructura la investigación no va a dar los frutos que todos requiren, debe existir integración entre el Estado, la Institución y la proyección de una vida mejor para los ciudadanos.

\section{Análisis de la categoría 4}

El tema de los perfiles ha sido muy discutido la última década. ¿Qué se espera de un profesional? ¿Cómo puede aportar un profesional al cambio social? ¿En qué medida la formación universitaria contribuye a esta formación de perfiles? Podemos partir de la idea de que el perfil de egreso de un estudiante universitario debe enforcarse en la multiplicidad; es decir, abarcar muchas posibilidades para de esa forma hacer frente a los problemas que la sociedad le muestra y entonces pueda salir airoso del mismo. Para los miembros de la Escuela de Frankfurt era estrictamente importante el hecho de la intervención de la ciencia en la mejora de los problemas de la sociedad; por tanto, debemos ser conscientes de la importancia de los perfiles de los estudiantes. Ahora, queda claro que, si un estudiante tiene un perfil acorde, es porque de una u otra manera ha tenido docentes con un perfil innovador, práctico y revelador de la problemática acuciosa de nuestra sociedad.

\section{Orientación metodológica}

El 56\% de los entrevistados asume que tanto el perfil epistemológico docente como el perfil del estudiante deben tener una orientación metodológica: actitud crítica ante los problemas de la sociedad, no tener prejuicios ni supuestos a favor o en contra de alguna teoría, predisposición a equivocarse en pos de mejorar en la búsqueda de soluciones relevantes. A manera de contraste cabe decir que Para Horkheimer era imposible separar las formas metodológicas del contenido del objeto de estudio, y tal vez por eso buscaba pensar en los problemas sociales que tenía a la vista y las formas de investigación que le correspondían en referencia mutua. Desde su punto de vista los hombres luchan y producen en torno a objetivos, necesidades y pasiones que sienten como propios. Explicar, valorar y criticar constituyen los 
núcleos epistemológicos articuladores con los cuales Horkheimer establece los andariveles de su interpretación histórica.

\section{Orientación innovadora}

Solo el 25\% de los entrevistados asume una orientación innovadora, destacan el hecho de arriesgarse por la ciencia, tratar de tener una buena comunicación entre pares. Lamentablemente, el porcentaje no ayuda a vislumbrar una mejora de posiciones en cuanto al avance de la ciencia. ¿Cómo optimizar el desarrollo de la epistemología ante los diversos problemas de la sociedad? En realidad, innovar implica procesos que no hemos logrado aún, somos una sociedad alienada que trata de escapar de la dialéctica del amo y el esclavo. ¿Cómo comprometernos? ¿Cómo innovar?

\section{Orientación teórica}

Solo el $18.75 \%$ de los entrevistados apostó por una respuesta de tipo teórica: comprender nuevos conocimientos, ser un ávido lector, tener compromiso con la investigación. Las características mencionadas son muy importantes, pero nos preocupa sobre manera el porcentaje de entrevistados que asume una respuesta como esta. Tal vez sea porque la mayoría de los docentes investigadores, o los estudiantes no cuentan con interés por el desarrollo de la ciencia, o porque piensan que investigar es demasiado absurdo, o porque no encuentran la sensación de disfrute antes esa aventura. Surge una serie ilimitada de preguntas ante un porcentaje como el que tenemos; ¿qué debemos hacer para ser mejores investigadores? ¿el perfil del egreso es igual al perfil del investigador?

\section{Conclusiones del análisis de la categoría 4:}

Luego de analizar las respuestas de los entrevistados en torno a la siguiente pregunta: el perfil epistemológico de todo estudiante y/o docente que desea ser investigador académico encontramos la siguiente tipificación en sus respuestas: Orientación metodológica, orientación innovadora y orientación teórica, Los entrevistados sugieren que la metodología podría ayudar para que el perfil de los estudiantes mejore con miras hacia el futuro. Otros señalan que lo mejor sería el tema de la innovación, puesto que a partir de la aplicación y nuevas estrategias se podrán verificar mejor hacia la mejora de los resultados de la invitación en el campo de las ciencias humanas. Finalmente, hay quien señala que sería mejor formular espacios teóricos par que de esa manera el perfil de los egresados mejores

\section{REFERENCIAS BIBLIOGRÁFICAS}

Durán Migliardi, M. (2018). Sociedad y Derecho: La influencia de la escuela de Frankfurt y su teoría crítica en los orígenes del pensamiento de Habermas. Universum (Talca), 33(1), 84-116. https://doi.org/10.4067/S0718$\underline{23762018000100084}$

Haidar, V. (2016). Sociedad de masas: lecturas procedentes del ordoliberalismo y de la Escuela de Frankfurt. Sociológica (México), 31(88), 29-64. http://www.scielo. org. $\mathrm{mx} / \mathrm{scielo}$. php? $\mathrm{script}=\mathrm{sci}$ arttextEpid =S018701732016000200029 \&lng $=$ esEnr $\underline{\mathrm{m}=\text { iso\&tlng }=}$ 
Rodríguez Reyes, A. (2017). De Frankfurt a rio cuarto: cuestiones para el diálogo analógico de la Teoría Crítica y la Filosofía de la Liberación. Cuadernos de Filosofía Latinoamericana, 37(115), 27-43. https://doi. org/10.15332/25005375/2945

Sembler, C. (2019). El socialismo de la Escuela de Frankfurt: Variaciones de Marx y la crítica del capitalismo. Izquierdas, 47, 167-183. https://doi.org/10.4067/ $\underline{\text { S0718-50492019000300167 }}$
Martin, J. L. (2013). El aporte de la Escuela de Fráncfort a la Pedagogía Crítica. El equilibrista. 1(1).

Navarro Salcedo, W. (2004). Teoría crítica de la educación. Paideia Surcolombiana, (11), 75-84. https:// doi.org/10.25054/01240307.1046

Wiggershaus, R. (2011). La Escuela de Fráncfort. F.C.E.

Fecha de recepción: 17/09/2021 Fecha de aceptación: 22/10/2021 
